\title{
Myocardial Injury and Myocarditis in SARS-CoV-2 Patients
}

\author{
Camelia Libenciuc', Răzvan-Andrei Licu1', Roxana Hodas', ${ }^{1,2}$ Monica Chitu²,3, Imre Benedek ${ }^{1,2,3}$ \\ 1 Clinic of Cardiology, Emergency Clinical County Hospital, Târgu Mureș, Romania \\ 2 "George Emil Palade" University of Medicine, Pharmacy, Science and Technology, Târgu Mureș, Romania \\ ${ }^{3}$ Center of Advanced Research in Multimodality Cardiac Imaging, CardioMed Medical Center, Târgu Mureș, Romania
}

\section{CORRESPONDENCE}

Răzvan-Andrei Licu

Str. Gheorghe Marinescu nr. 50

540136 Târgu Mureş, Romania

Tel: +40 265212111

E-mail: licu.razvan@yahoo.com

\section{ARTICLE HISTORY}

Received: June 20, 2020

Accepted: July 19, 2020
Camelia Libenciuc • Str. Gheorghe Marinescu nr. 50, 540136 Târgu Mureș, Romania. Tel: +40 265-212.111, E-mail: camelialibenciuc@gmail.com

Roxana Hodas • Str. Gheorghe Marinescu nr. 38 540139 Târgu Mureș, Romania. Tel: +40 265215551 E-mail: roxana.hodas@yahoo.ro

Monica Chitu • Str. Gheorghe Marinescu nr. 38 540139 Târgu Mureș, Romania. Tel: +40 265215551 , E-mail: iulia.chitu@yahoo.com

Imre Benedek • Str. Gheorghe Marinescu nr. 38 540139 Târgu Mureș, Romania. Tel: +40 265215551 E-mail: imrebenedek@yahoo.com

\begin{abstract}
Myocarditis is one of the relatively common complications of respiratory infection with SARSCoV-2. As several patients confirmed with the new SARS-CoV-2 are known with cardiovascular disease (CVD) and data from the literature show negative prognosis and a higher risk of complications, this subgroup of subjects represents a particular situation. Therefore, an adequate understanding of the mechanisms involved in myocardial injury and interaction between COVID-19 and CVD is essential for optimal further management. Studies have proved that in COVID-19 patient myocarditis is determined via three pathological mechanisms of cardiomyocyte injury: direct viral cell entry and binding to ACE2, vasculitis-mediated injury, and systemic inflammatory response leading to pro-inflammatory cytokine discharge. Studies show that the incidence of myocarditis in patients with SARS-CoV-2 is relatively low, $4.8 \%$, but myocardial damage occurs in more than $25 \%$ of critical cases in the form of acute fulminant myocarditis with severe hemodynamic degradation, or develops when the severity of SARS-CoV-2 infection intensifies. The mortality rate in myocarditis from COVID-19 infection ranges between $50-70 \%$, with poorer prognosis and a higher risk of complications in CVD patients. As in all of these cases increased troponin and natriuretic peptide levels proved to be a negative prognostic factor, for risk stratification and prompt treatment, cardiac biomarkers should be evaluated in all patients with COVID-19.
\end{abstract}

Keywords: SARS-CoV-2, myocarditis, ACE2 receptors, cardiovascular disease, cardiac biomarkers

\section{SARS-COV-2 - A GLOBAL OUTBREAK}

Coronaviruses represent an RNA genome-based group of viruses that cause disease particularly in animals and birds. At this moment, there are only seven types of this viral group known to affect people, including SARS-CoV-2, which is believed to have originated in Wuhan, China. SARS-CoV-2 causes mainly severe acute respiratory distress, but besides pulmonary damage, other organs, such as the heart, kidney and liver, are also injured. ${ }^{1}$ A particular situation is represented by the worsening condition of cardiac patients. As several patients confirmed with the new coronavirus are known with cardiovascular diseases (CVD), an adequate understanding of the interaction between COVID-19 and cardiovascular comorbidities is essential for the optimal management of these 
patients. The most common cardiovascular complications given by COVID-19 are myocarditis, acute myocardial infarction, exacerbation of chronic heart failure, and pulmonary thromboembolism. ${ }^{2}$ Myocarditis proved to be a relatively common complication of SARS-CoV-2, with the underlying pathological mechanism consisting of cardiomyocyte injury determined by systemic inflammation. ${ }^{3}$ It is estimated that over $40 \%$ of SARS-CoV- 2 deceased patients presented myocardial lesions. The first confirmed case of COVID-19 in a cardiac patient was on December 8, 2019, in the Hubei province of China. ${ }^{4}$

\section{CARDIOVASCULAR COMORBIDITIES IN COVID-19 PATIENTS}

According to a recent meta-analysis conducted on 53,000 patients who have been positively confirmed with SARSCoV-2, cardiovascular risk factors and comorbidities (arterial hypertension, diabetes, various CVDs) were present in $30 \%$ of subjects. ${ }^{5}$ Moreover, studies have shown that the mortality rate in patients with pre-existing heart disease was $2.3 \%$ in 1,023 deceased patients out of 44,672 confirmed positive. Several recent reports indicate that $28 \%$ of hospitalized patients presented myocardial impairment, a fact also confirmed by the presence of viral RNA in $35 \%$ of the autopsied hearts. ${ }^{6}$ Myocardial damage occurs in the form of acute fulminant myocarditis, with severe hemodynamic degradation or development as the severity of SARS-CoV-2 infection intensifies. ${ }^{7}$

Data available from the literature show that SARS-CoV-2 is responsible for direct myocardial injury by penetrating into human cells and binding to angiotensin-converting enzyme 2 (ACE2), which is highly expressed especially in the lungs and the heart. This leads to an alteration of ACE2 signaling pathways and eventually triggers acute pulmonary and myocardial lesions. ${ }^{8}$ The second mechanism involved may be vasculitis mediated by infection. ${ }^{9}$ In addition to the heart and lungs, ACE2 receptors are also highly expressed in arterial and venous endothelial cells. Therefore, myocardial injury occurs either by direct viral entry into myocardial endothelial cells, or by triggering an indirect immunological response leading to a hypersensitivity reaction, which suggests an intrinsic susceptibility of the heart to SARS-CoV-2. ${ }^{10}$ The third mechanism involved in the appearance of myocarditis in COVID-19 patients consists is the presence of an acute systemic inflammatory response leading to large pro-inflammatory cytokine discharge into the circulation, substances that can induce damage to several organs and can eventually lead to multiple organ dysfunction syndrome (MODS). ${ }^{11}$

\section{MYOCARDITIS CAUSED BY THE NOVEL CORONAVIRUS}

One of the first studies conducted in China on 84 hospitalized patients found that the incidence of myocarditis in patients with SARS-CoV-2 is relatively low, being reported at $4.8 \%$. From the 84 admitted patients, 13 had indicators of myocardial lesion and abnormal ECG, 8 patients were in serious condition, and 5 had uncertain diagnosis. ${ }^{12}$ Reported ECG abnormalities included bradycardia or sinus tachycardia, atrioventricular block, modified $\mathrm{T}$ waves, or abnormal Q waves. In evolution, after the decrease of inflammatory markers, troponin levels and ECG returned to normal. The results of the study showed that the level of cardiac enzymes was significantly correlated with the level of C-reactive protein (CRP), and the ECG changes with the level of procalcitonine (PCT) and the number of lymphocytes. However, at that time, no correlations were identified between cardiac troponin I (cTnI) and the level of inflammatory or lymphocyte markers. ${ }^{13}$ Further cohort studies conducted in China on 341 patients estimated that myocardial lesion occurs in $7 \%$ to $17 \%$ of hospitalized patients, more common in patients admitted to intensive care (22.2\% vs. $2.01 \%)$, and among subjects who have died (59\% vs. $1 \%)^{14}$

Kun-Long Ma et al. reported alarming mortality rates in patients with myocarditis from COVID-19 infection, of up to $50-70 \% .{ }^{15}$ The first case of fulminant myocarditis was reported in a 63-year-old male patient, hospitalized with a fever of $39.3^{\circ} \mathrm{C}$, chest pain, and shortness of breath. He was a chronic tobacco user with an allergic cough for five years, but with no history of heart disease. Laboratory data showed high levels of troponin I (11.37 g/L) and myoglobin $(390.97 \mathrm{pg} / \mathrm{nL})$, and n-terminal brain natriuretic peptides (NTBNP) levels of $22,600 \mathrm{pg} / \mathrm{mL}$. The ECG showed sinus tachycardia, without ST segment elevation. Echocardiography revealed a severely altered left ventricle ejection fraction of $32 \%$, with a dilated left ventricle $(61 \mathrm{~mm})$ and a pulmonary artery systolic pressure of $44 \mathrm{mmHg} .{ }^{16} \mathrm{An}$ other study, which included 187 patients, confirmed that the presence of myocardial lesion is in strong correlation with mortality rates in COVID-19, while the prognosis of patients with CVD but without myocarditis is relatively favorable. From 187 patients with SARS-CoV-2, 27.8\% have developed myocardial lesions, followed by cardiac dysfunction and arrhythmias. SARS-CoV-2-associated myocarditis occurred in 5 of the first 41 positive patients from Wuhan. In this study, 4 out of 5 patients with myocardial lesions were admitted to intensive care, which shows the severity of infection in these patients. ${ }^{17}$ 


\section{MYOCARDIAL INJURY-RELATED DEATHS IN COVID-19 PATIENTS}

Mortality in patients with SARS-CoV-2 was associated with increased troponin levels of above the 99th percentile of the normal upper limit in $8-12 \%$ of positive patients. ${ }^{18}$ Recently, it has been observed that $20-30 \%$ of patients with severe form of COVID-19 infection presented very high levels of cardiac troponins. ${ }^{19}$ Hospitalized patients, confirmed positively with the new coronavirus but with a normal troponin level, presented a better prognosis, with a mortality between 5-13\%, compared to those with high levels of cardiac biomarkers, who had a mortality rate of $50 \%$. The mechanism by which troponin levels are elevated in SARS-CoV-2 infection has been linked to direct viral infection of myocytes and triggering the cascade of pro-inflammatory cytokines. ${ }^{20}$ Endomyocardial biopsy, the gold standard for the diagnosis of myocarditis, could be useful in these cases, but there is no standardized procedure in COVID-19 cases yet..$^{21}$ In a recent study, out of 150 patients with COVID-19, 68 subjects have died, of which $33 \%$ had been diagnosed with acute myocarditis, while $7 \%$ of deaths were attributed to myocarditis associated with circulatory failure. ${ }^{22}$ This information reinforces the statement that myocarditis becomes a complication of SARS-CoV-2 that is difficult to manage and treat.

In other reports, fulminant myocarditis was strongly associated with very high viral load. In these cases, the autopsy results showed mononuclear inflammatory infiltration of the myocardial tissue. ${ }^{13}$ Myocardial involvement in COVID-19 was found in more than $20 \%$ of patients and was linked to increased mortality. Furthermore, an association of low-grade myocardial inflammation with cardiogenic shock has been found. ${ }^{13}$

An interesting particularity found in patients with SARS-CoV-2 is the presence of myocardial injury due to Takotsubo syndrome. It was reported in a 43-year-old woman, who presented to the emergency room after three days from onset of chest pain and dyspnea, with no significant medical history. After treatment with hydroxychloroquine and lopinavir/ritonavir, within a few days left ventricular ejection fraction had returned to normal (65\%), with a normalized ECG tracing, and troponin and CRP levels within normal range. The underlying mechanism for the occurrence of Takotsubo remains uncertain. ${ }^{9}$

Corticosteroids and immunoglobulins represent the supportive treatment options available in cases of myocarditis associated with SARS-CoV-2. Until this moment, treatment with methylprednisolone $(200 \mathrm{mg} /$ day for 4 days) proved to decrease inflammation, while immuno- globulins regulate immunity. In a study conducted by Xiong et al., after a week, the chest X-ray showed a normalsized heart, and the echocardiography revealed normal heart function and cavity size. Incidentally, the markers of myocardial lesions decreased significantly. ${ }^{24}$

\section{CONCLUSIONS}

Cardiovascular involvement is common in SARS-CoV-2 infection. Thus, there is an important need to identify patients at increased risk of death and offer supportive treatment. A relatively common complication of SARS-CoV-2 infection includes acute myocarditis as a first clinical manifestation. Alarming mortality rates have been associated with myocardial damage that occurs in the form of acute fulminant myocarditis, with severe hemodynamic degradation. Moreover, the presence of myocardial damage in COVID-19 patients with cardiovascular comorbidities is associated with high mortality and morbidity. As in all of these cases increased troponin and natriuretic peptide levels proved to be a negative prognostic factor, cardiac biomarkers should be evaluated and used for risk stratification of patients with confirmed SARS-CoV-2 infection.

\section{CONFLICT OF INTEREST}

Nothing to declare.

\section{ACKNOWLEDGEMENT}

This research was supported via the research grant no. 103544/2016 - PLaqueIMAGE, contract number 26/01.09.2016, financed by the Romanian Ministry of European Funds, the Romanian Government and the European Union.

\section{REFERENCES}

1. Basu-Ray I, Almaddah NK, Adeboye A, Soos MP. Cardiac Manifestations Of Coronavirus (COVID-19). In: StatPearls. Treasure Island (FL): StatPearls Publishing, 2020

2. Bansal M. Cardiovascular disease and COVID-19. Diabetes Metab Syndr. 2020;14:247-250.

3. Hulot JS. COVID-19 in patients with cardiovascular diseases. Arch Cardiovasc Dis. 2020;113:225-226.

4. Zhu H, Rhee JW, Cheng P, et al. Cardiovascular Complications in Patients with COVID-19: Consequences of Viral Toxicities and Host Immune Response. Curr Cardiol Rep. 2020;22:32.

5. Clerkin KJ, Fried JA, Raikhelkar J, et al. COVID-19 and Cardiovascular Disease. Circulation. 2020;141:1648-1655

6. Zheng YY, Ma YT, Zhang JY, Xie X. COVID-19 and the cardiovascular system. Nat Rev Cardiol. 2020;17:259-260.

7. Xu Z, Shi L, Wang Y, et al. Pathological findings of COVID-19 associated with acute respiratory distress syndrome. Lancet Respir Med. 2020;8:420422 
8. Chen L, Li X, Chen M, Feng Y, Xiong C. The ACE2 expression in human heart indicates new potential mechanism of heart injury among patients infected with SARS-CoV-2. Cardiovasc Res. 2020;116:1097-1100.

9. Driggin E, Madhavan MV, Bikdeli B, et al. Cardiovascular Considerations for Patients, Health Care Workers, and Health Systems During the COVID-19 Pandemic. J Am Coll Cardiol. 2020;75:2352-2371.

10. Atri D, Siddiqi HK, Lang JP, Nauffal V, Morrow DA, Bohula EA. COVID-19 for the Cardiologist: Basic Virology, Epidemiology, Cardiac Manifestations, and Potential Therapeutic Strategies. JACC Basic Trans/ Sci. 2020;5:518-536.

11. Guzik TJ, Mohiddin SA, Dimarco A, et al. COVID-19 and the cardiovascular system: implications for risk assessment, diagnosis, and treatment options. Cardiovasc Res. 2020;116:1666-1687.

12. Lippi G, Lavie CJ, Sanchis-Gomar F. Cardiac troponin I in patients with coronavirus disease 2019 (COVID-19): Evidence from a meta-analysis. Prog Cardiovasc Dis. 2020;63:390-391.

13. Hu H, Ma F, Wei X, Fang Y. Coronavirus fulminant myocarditis treated with glucocorticoid and human immunoglobulin. Eur Heart J. 2020

14. Maisch B. SARS-CoV-2 as potential cause of cardiac inflammation and heart failure. Is it the virus, hyperinflammation, or MODS? SARS-CoV-2 als mögliche Ursache für Myokarditis und Herzinsuffizienz. Ist es das Virus, Hyperinflammation oder MODS? Herz. 2020;45:321-322.

15. Deng Q, Hu B, Zhang $Y$, et al. Suspected myocardial injury in patients with COVID-19: Evidence from front-line clinical observation in Wuhan, China. Int J Cardiol. 2020;311:116-121.
16. Sala S, Peretto G, Gramegna M, et al. Acute Myocarditis Presenting as a Reverse Takotsubo Syndrome in a Patient With SARS-CoV-2 Respiratory Infection. Eur Heart J. 2020;41:1861-1862.

17. Kochi AN, Tagliari AP, Forleo GB, et al. Cardiac and arrhythmic complications in patients with COVID-19. Cardiovasc Electrophysiol. 2020;31:1003-1008.

18. Chen C, Zhou Y, Wang DW. SARS-CoV-2: a potential novel etiology of fulminant myocarditis. Herz. 2020;45:230-232.

19. Babapoor-Farrokhran S, Gill D, Walker J, Rasekhi RT, Bozorgnia B Amanullah A. Myocardial injury and COVID-19: Possible mechanisms. Life Sci. 2020;253:117723

20. Huang C, Wang Y, Li X, et al. Clinical features of patients infected with 2019 novel coronavirus in Wuhan, China. Lancet. 2020;395:497-506.

21. Ozieranski K, Tyminska A, Caforio ALP. Clinically suspected myocarditis in the course of coronavirus infection. Eur Heart J. 2020;41:2118-2119.

22. Oudit GY, Kassiri Z, Jiang C, et al. SARS-coronavirus modulation of myocardial ACE2 expression and inflammation in patients with SARS. Eur J Clin Invest. 2009;39:618-625.

23. Augoustides JG. Cardiovascular Consequences and Considerations of Coronavirus Infection - Perspectives for the Cardiothoracic Anesthesiologist and Intensivist During the Coronavirus Crisis. J Cardiothorac Vasc Anesth. 2020;S1053-0770(20)30315-3.

24. Xiong TY, Redwood S, Prendergast B, et al. Coronaviruses and the cardiovascular system: acute and long-term implications. Eur Heart J. 2020;41:1798-1800. 